\title{
Risks and Benefits of the Intercostal Approach for Percutaneous Nephrolithotripsy
}

\author{
Erich K. Lang, Raju Thomas, Rodney Davis, Ivan Colon, Wellman Cheung, Erum Sethi, Ernest \\ Rudman, Amer Hanano, Leann Myers, Alexander Kagen
}

Departments of Radiology (EKL, ES, ER, AH, AK) and Urology (IC), SUNY Downstate School of Medicine, Brooklyn, New York, Department of Urology (RT, RD), Tulane Health Science Center, New Orleans, Louisiana, Department of Biostatistics (LM), Tulane School of Tropical Medicine, New Orleans, Louisiana, and Department of Radiology (EKL), Johns Hopkins Medical Institutions, Baltimore, Maryland, USA

\begin{abstract}
Objective: The objective of our retrospective study was to provide evidence on the efficacy of the intercostal versus subcostal access route for percutaneous nephrolithotripsy.

Materials and Methods: 642 patients underwent nephrolithotomy or nephrolithotripsy from 1996 to 2005 . A total of 127 had an intercostal access tract (11th or 12th); 515 had a subcostal access tract.

Results: Major complications included one pneumothorax (1.0\%), one arterio-calyceal fistula (1.0\%) and three arteriovenous fistulae (2.7\%) for intercostal upper pole access; two pneumothoraces (1.7\%), one arteriovenous fistula (1.0\%), one pseudoaneurysm (1.0\%), one ruptured uretero-pelvic junction (1.0\%), 4 perforated ureters (3.4\%) for subcostal upper pole access; one hemothorax (1.6\%), one colo-calyceal fistula (1.6\%), one AV fistula (1.6\%), and two perforated ureters (3.2\%) with subcostal interpolar access. Diffuse bleeding from the tract with a subcostal interpolar approach occurred $3.2 \%$ of the time compared with $2.4 \%$ with a lower pole approach. Staghorn calculi demonstrated similar rates of complications. Conclusion: Considering the advantages that the intercostal access route offers the surgeon, it is reasonable to recommend its use after proper pre-procedural assessment of the anatomy, and particularly the respiratory lung motion.
\end{abstract}

Key words: kidney; calculi; lithotripsy; nephrostomy, percutaneous; thorax; complications Int Braz J Urol. 2009; 35: 271-83

\section{INTRODUCTION}

Percutaneous nephrolithotomy and nephrolithotripsy have emerged as procedure of choice in the management of staghorn calculi and in patients presenting with a large stone burden $(1,2)$. Extracorporeal shockwave lithotripsy (SWL) is transposed to problems of potential retained and residual fragments, with subsequent "steinstrasse" formation (3). Conversely, percutaneous techniques have attained a stone free status in up to $98.3 \%$ of targeted renal stones (4).
Access via the superior posterior calyx offers optimal exposure to staghorn calculi as well as multiple calculi in the superior and inferior calyceal groups, renal pelvis, and upper ureter, and is therefore generally preferred by urologists (Figures-1 and 2). However, prior publications on the subject have suggested a higher rate of complications with 11th and 12th intercostal approach (5-8). The purpose of our study was to compare the rates of complications via the 11th and 12th intercostal upper pole approaches with those via subcostal access routes. In addition, we will examine the indications for upper pole versus 
subcostal access routes for different stone locations as well as the relevant anatomy that may increase the rate of complications for a given access route (9-12).

\section{MATERIALS AND METHODS}

The study-population consisted of 642 patients, 367 male, 275 female, ages 15-91 years, 46 years mean age, who underwent percutaneous nephrolithotripsy and nephrolithotomy from 1996 to 2005 at the Medical Center of Louisiana, New Orleans V.A. Hospital, Tulane Health Science Center and SUNY, Downstate Medical School, Brooklyn, N.Y. selection criteria for use of percutaneous nephrolithotripsy and nephrolithotomy were staghorn calculi involving the superior calyceal group, a stone mass greater then $2500 \mathrm{~mm}^{2}$, calculi in superior calyceal group as well as pelvis and upper ureter, stones in high lying kidneys and sometimes horseshoe kidneys as well as failures of SWL. A total of 127 had an intercostal (11th or 12th rib, 73 left and 54 right kidney); and 515 had a subcostal access approach. In 133 patients, a second and third access tract became necessary to reach all stone bearing areas.

In 255 patients, the calculi were classified as staghorn, in 160 as multiple, and in 227 as single calculi. Non-contrast computed tomography (CT) examinations were reviewed to establish the location of calculi and determine an optimal approach. An intercostal approach was favored for staghorn calculi, multiple calculi located in the superior posterior, anterior and posterior inferior calyceal group, pelvis and uretero-pelvic junction (UPJ); a subcostal approach for solitary calculi in the anterior or posterior inferior, anterior and posterior interpolar calices and pelvis or combinations thereof. For calculi inaccessible from the primary access tract, a "Y" tract or a new percutaneous access tract was developed. Ninety percent of the intercostal procedures were performed in the last 5 years. Ninety percent of all intercostal and 50\% of all subcostal access procedures were performed by a senior interventional radiologist (with more than 30 years of experience); the remainder by 2 senior interventional radiologists (with 5 and 10 years experience respectively) well beyond the learning curve and always in conjunction with a senior urologist.
Informed consent was obtained in all procedures. The respective institutional review committees had approved these procedures.

In order to establish a straight tract from the skin to the posterior superior calyx, infundibulum, and renal pelvis, triangulation was used with the aid of CT, preferentially 3-dimensional CT reconstructions (12,13), Figure-2. The angle of entry approximates 30 degrees, with straight-line continuation through the infundibulum into the pelvis in an anterior-inferior-medial direction (the renal pelvis being approximately $1.5 \mathrm{~cm}$ anterior to the posterior superior calyx). This trajectory takes advantage of the avascular zone of Brodel. The patients are then placed on the fluoroscopic table, with the back and flank prepped and draped using standard surgical technique. Meticulous assessment of diaphragmatic excursion in inspiration and expiration was obtained to establish a safe and appropriate point of entry, avoiding the pleura and lung. Puncture is carried out in mid-expiration to minimize risk of puncture of the

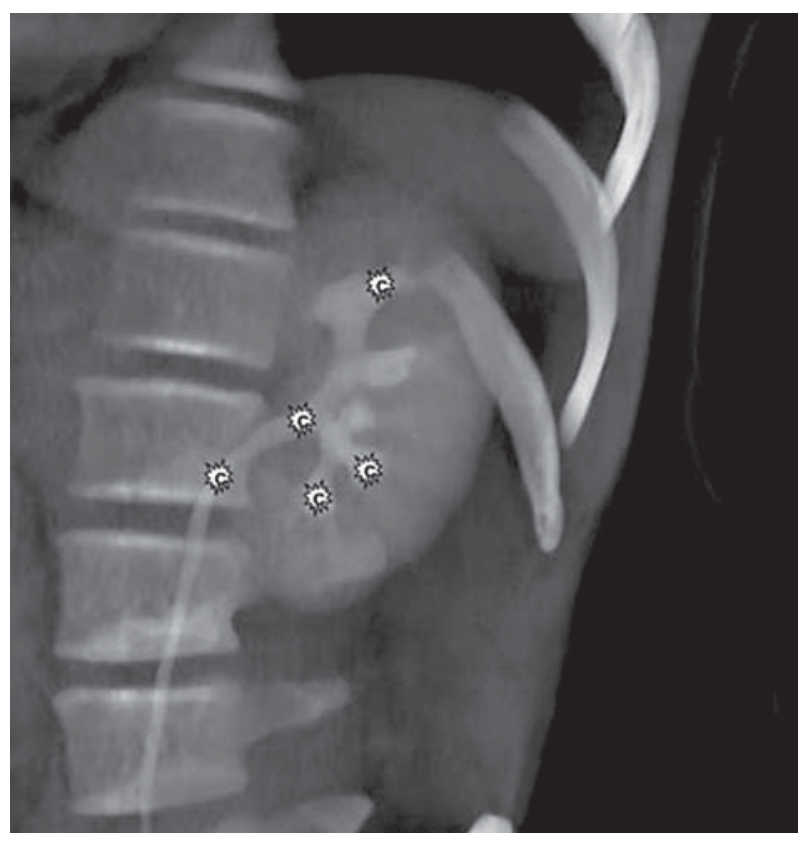

Figure 1 - Three-dimensional volume rendered oblique coronal reformatted image with $10 \%$ gray demonstrating the accessibility of stone bearing locations when the intercostal approach is used for percutaneous removal of calculi from the posterior superior calyx, pelvis, posterior and anterior inferior calyx, ureter and of staghorn calculi. $c=$ calculus. 
lung even though the needle tract may traverse the lowermost pleura $(11,12,14)$. Infusion of a $200 \mathrm{~mL}$ bolus of N-Saline into the pleural space was another modification used to prevent puncture of the lung. Because of the known increased risk of pneumothorax or even calico-pleural fistulae access via the 11th interspace was avoided whenever possible $(4,8,13$ 16).

The initial access is carried out in the Interventional Radiology Unit under fluoroscopic control. Local anesthesia, complemented by conscious sedation, was routinely used. Thereafter a 22 -gauge needle is advanced blindly into the kidney until urine is aspirated, indicating puncture of the collecting system. Approximately $8 \mathrm{~mL}$ of $50 \%$ dilute nonionic contrast medium are then injected to outline the collecting system. Under biplanar fluoroscopic (or rarely $\mathrm{CT}, \mathrm{n}=14$, or ultrasound, $\mathrm{n}=5$ ) guidance, the center of the fornix of the targeted calyx is accessed with an 18-gauge diamond tip needle (which is the most peripheral point of the calyx). A 0.038 inch guide wire is then advanced into the

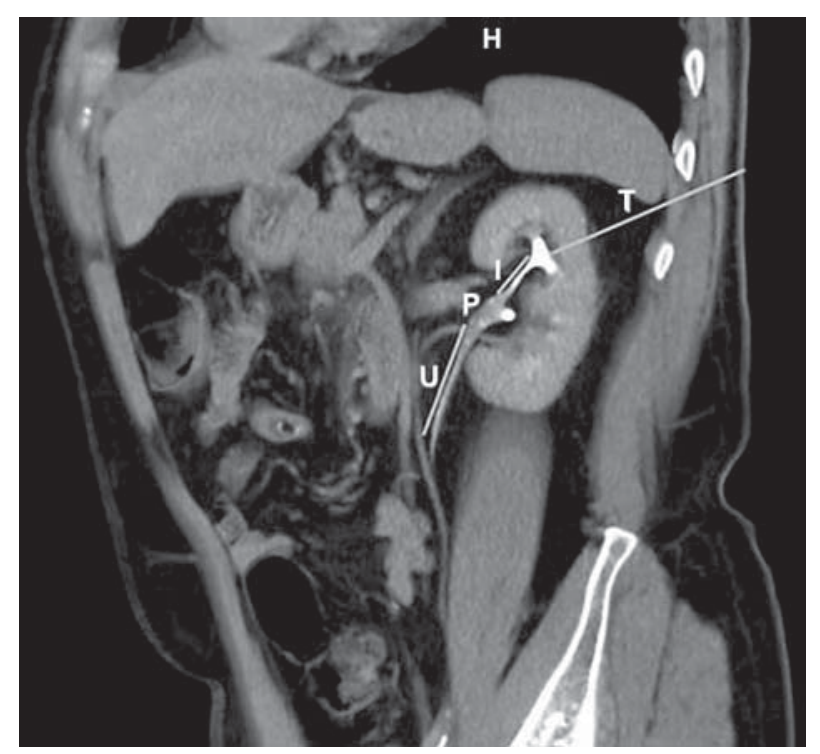

Figure 2 - Three-dimensional oblique coronal multi-planar reformatted image demonstrating a relatively straight path from the point of entry, through the infundibulum and renal pelvis and to the ureter, afforded by an intercostal posterior upper polar calyceal access, forming only a small angle with the renal axis. $T=$ access tract, $I=$ infundibulum, $P=$ renal pelvis, $U=$ ureter. (courtesy Urology Elsevier) renal pelvis, the tract dilated with 6 and $8 \mathrm{~F}$ Teflon dilators, and finally a renal curve Cobra 2 Catheter (Boston Scientific, Boston, MA, USA) is advanced into the pelvis and ureteropelvic junction is engaged. A glidewire (Boston Scientific, Natick, MA, USA) is then advanced under fluoroscopic control into the bladder. Finally, it is replaced through an exchange sheath with super-stiff 0.038 Amplatz wires (1st working wire in pelvis, and a second safety wire in bladder). At this point, the patient is transferred to the operating room and the procedure continued under general anesthesia. The tract is dilated with a high pressure Pathway balloon (Boston Scientific, Natick, MA, USA) and a 28 - 32F Amplatz sheath (Bard, Covington, GA, USA) is advanced over the inflated balloon into the desired calyx. The Amplatz sheath serves to tamponade the tract, keeping the pressure in the accessed system at a low of $16 \mathrm{~mm} \mathrm{H}_{2} \mathrm{O}$ and allows access for the rigid nephroscope. Percutaneous stone removal under general anesthesia is now carried out. At the completion of the procedure, all patients had a 22-G nephrostomy tube and double "J" ureteral stent inserted.

\section{ANATOMIC CONSIDERATIONS}

For safe access via the 11th or 12th interspace, location of the posterior costo-phrenic sulcus particularly, on the left side should be established in both inspiration and expiration by fluoroscopy (12). In most patients, a viable trajectory to the posterior superior pole calyx can be achieved that does not violate the pleural space or lung (Figure-2). Retro-renal position of the left colon is one condition occurring in approximately $10 \%$ of prone patients (12). This could preclude access via a 11th or 12th intercostal approach in those select patients. In some patients, a large spleen can provide a challenge.

Access to the posterior upper pole calyx affords an almost straight path to the renal pelvis, upper ureter and both anterior and posterior inferior calyceal groups (Figures-1 to 4). Even the posterior interpolar calyx may be accessible via this path without significant angulation. The potential to advance the Amplatz sheath or nephroscope in a straight path from the posterior upper pole calyx into the renal 

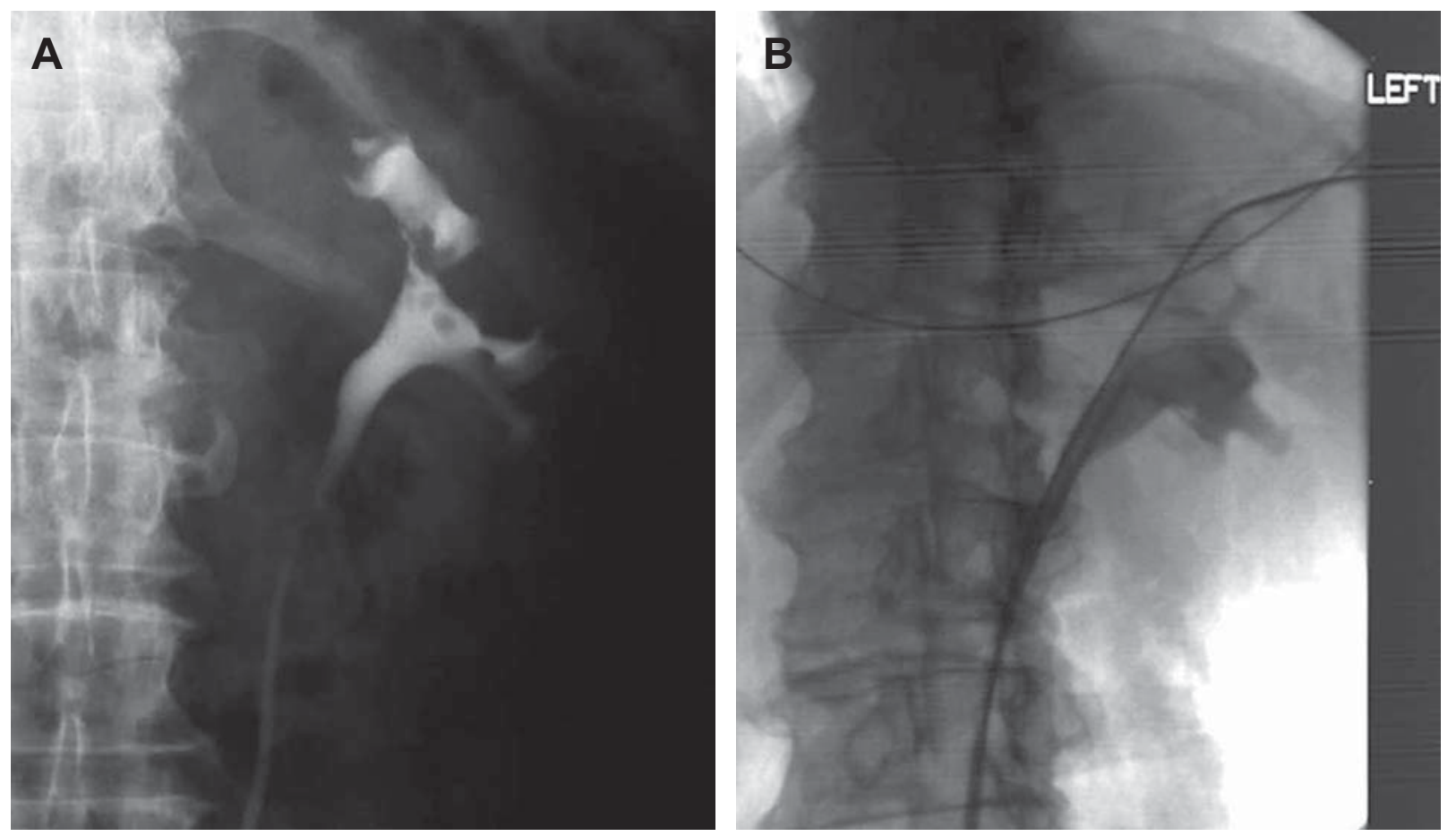

Figure 3 - A) Patient with infundibular transitional cell carcinoma. B) The posterior superior calyx was entered via an intercostal approach for tumor ablation. Note the option offered by the straight trajectory to inspect the renal pelvis, inferior anterior and posterior calices and ureter.

pelvis, upper ureter, and anterior and posterior inferior calyceal groups is of great advantage to the urologist, and reduces the propensity for injury to the peri-infundibular venous plexus if an angulation of the tract is necessary to reach the stone-bearing region $(15,16)$. While the infundibulum of the superior calyceal group tends to be longer, the vascularity of the peri-papillary and peri-infundibular plexus is less prominent than in the mid calyceal (interpolar calyceal) group (11). Access via the posterior superior calyceal group makes calculi in the renal pelvis, upper ureter, and anterior and posterior inferior calyceal groups accessible, and thus makes this an almost universal access route. Only the superior anterior calyceal group and anterior and sometimes posterior interpolar calyceal groups cannot be reached easily via this entry and hence may mandate separate punctures and access routes if calculi are harbored in these regions $(5,15)$. Moreover, access from the superior posterior calyceal group creating a straight path to the pelvis, upper ureter and inferior calyceal group reduces injury to renal parenchyma by the Amplatz sheath or nephroscope during respiratory excursion $(12,14)$.

\section{RESULTS}

To provide access for nephrolithotripsy of calculi in the posterior superior calyx, the intercostal access route was chosen in 111 patients, and the subcostal route in 119 patients (Table-1). A total of 134 of these patients were treated for a staghorn calculus, another 96 for at least one calculus lodged in the superior calyx (Table-2). To attain a near stone free status, the mid calyceal group had to be accessed secondarily in 44 patients with staghorn calculi (for dendritic stones or residual debris) and in 11 others (for otherwise inaccessible stones or debris); as well as the inferior calyx in 15 patients with staghorn calculi and 16 patients with residual calculi or debris (Table-2). Patients with residual or otherwise inaccessible calculi mandated access to the mid calyx in 


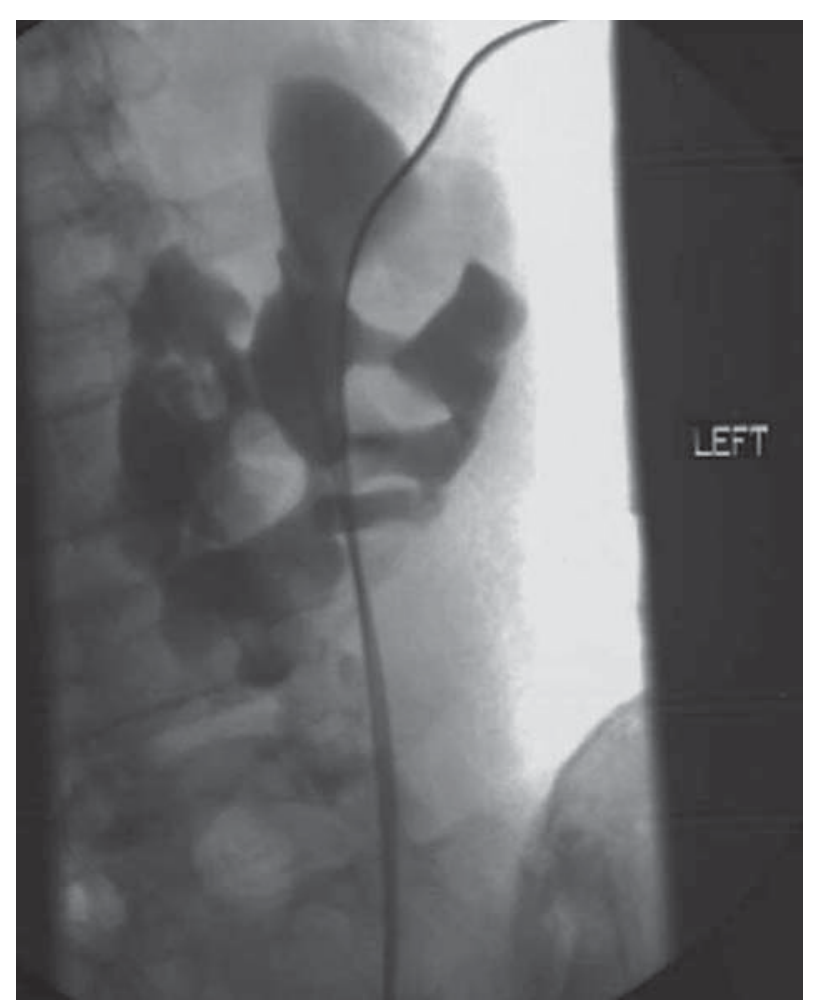

Figure 4 - The superior posterior calyx of a horseshoe kidney is easily accessed via an intercostal approach. Again, note the straight trajectory to the renal pelvis, inferior calices and ureter.

75 patients (12 via intercostal and 63 via subcostal approach) and in 31 to the inferior calyx (2 via intercostal and 29 via subcostal approach) (Table-2). An abnormal high location of the kidney mandated access via the intercostal route in 2 patients, with calculi in the mid calyx and 2 in the lower pole calyx (Table2). Overall, the midcalyx was directly accessed via intercostal route in 12 patients and via a subcostal approach in 63 patients; the lower pole calyx via an intercostal route in 4 patients and via a subcostal approach in 333 patients (Table-1).

The major complications we experienced were septic shock and effects to the vascular system, collecting system, and lungs. Access to the upper pole by the intercostal route resulted in 1 pneumothorax, 1 arterio-calyceal fistula and $3 \mathrm{AV}$ fistulae in 111 patients (Table-3). Via a subcostal access route, we recorded 2 pneumothoraces, $1 \mathrm{AV}$ fistula, 1 pseudoaneurysm, 1 ruptured UPJ, and 4 perforated ureters in 119 patients (Table-3). The ratio of complication to no complication was significant $(p=0.0395)$. In the same group of patients, we experienced 7 minor complications in the intercostal access group and 23 in the subcostal access group (Table-4). A high incidence of atelectasis $(\mathrm{n}=$ 13) in the subcostal access group as well as a relatively high need for blood transfusion $(n=2)$ should be noted (Table-4). For intercostal access to the mid-calyceal group, we recorded no major complications. However, for subcostal access, the rate was exceedingly high (5 in 63 patients, $7.8 \%$ ). We recorded 1 hemothorax, $1 \mathrm{AV}$ fistula, 1 colo-calyceal fistula and 2 perforated ureters (Table-3). Among minor complications, the need for blood transfusions in the intercostal access group was high (2 in 12 patients, 16.7\%). Minor complications in the subcostal entry group were high (12 in 63 patients, 19\%); again blood transfusions were among the most frequent of the minor complications, occurring in 4 patients $(6.3 \%$, Table- 4$)$. We experienced no major complications with entry into the lower poles via an intercostal route, however via a subcostal route there were 5 major complications in 333 patients. Septic shock in 2 patients $(0.6 \%)$ is noteworthy, since it is easily avoidable (Table-3). Of all minor complications, diffuse tract bleeding oc-

Table 1 - Primary and secondary access for nephrolithotripsy.

\begin{tabular}{|c|c|c|c|c|c|c|}
\hline \multirow[b]{2}{*}{ Location } & \multicolumn{3}{|c|}{ Intercostal } & \multicolumn{3}{|c|}{ Subcostal } \\
\hline & UP & MP & LP & UP & MP & LP \\
\hline Primary Access & 111 & 12 & 4 & 119 & 18 & 304 \\
\hline Secondary Access & & 10 & 2 & & 45 & 29 \\
\hline
\end{tabular}

$U P=$ upper pole $; M P=$ mid pole $; P=$ lower pole. 
Table 2-Access by location of calculi.

\begin{tabular}{|c|c|c|c|c|c|c|}
\hline \multirow[b]{2}{*}{ Location } & \multicolumn{3}{|c|}{ Intercostal } & \multicolumn{3}{|c|}{ Subcostal } \\
\hline & UP & MP & LP & UP & MP & LP \\
\hline \multicolumn{7}{|l|}{ Staghorn calculus } \\
\hline Primary access & 102 & 1 & 1 & 32 & 12 & 48 \\
\hline Secondary access & & 9 & 1 & & 35 & 14 \\
\hline \multicolumn{7}{|l|}{ Multiple calculus } \\
\hline Primary access & 8 & & & 41 & 4 & 82 \\
\hline Secondary access & & & & & 10 & 15 \\
\hline \multicolumn{7}{|l|}{ Single calculus } \\
\hline Primary access & 1 & 1 & 1 & 46 & 2 & 174 \\
\hline Secondary access & & 1 & 1 & & & \\
\hline
\end{tabular}

$U P=$ upper pole; $M P=$ mid pole $[L=$ lower pole.

curring in 8 patients $(2.4 \%)$, which deserves special attention (Figure-5). In the subgroup of staghorn calculi, 4 major vascular complications occurred in 102 intercostal accesses to the upper pole (4\%, Table$5)$. An even higher incidence of major complications occurred when subcostal access was provided (4 in 32 patients, $12.5 \%$ ). The incidence of complications with mid-polar subcostal route access is again very high (5 in 47), Table-5.

\section{COMMENTS}

The literature reports a stone-free status attainable by percutaneous nephrolithotripsy (PCNL)

Table 3 - Major complications at various access sites.

\begin{tabular}{|c|c|c|c|c|c|c|}
\hline \multirow[b]{2}{*}{ Location } & \multicolumn{3}{|c|}{ Intercostal } & \multicolumn{3}{|c|}{ Subcostal } \\
\hline & UP & MP & LP & UP & MP & LP \\
\hline No. Punctures & 111 & 12 & 4 & 119 & 63 & 333 \\
\hline \multicolumn{7}{|l|}{ Complications } \\
\hline Pneumothorax & $1(1 \%)$ & & & $2(1.7 \%)$ & & \\
\hline Hemothorax & & & & & $1(1.6 \%)$ & \\
\hline Colo-caliceal fistula & & & & & $1(1.6 \%)$ & \\
\hline Arterio-caliceal fistula & $1(1 \%)$ & & & & & \\
\hline AV fistula & $3(2.7 \%)$ & & & $1(1 \%)$ & $1(1.6 \%)$ & \\
\hline Pseudoaneurysm & & & & $1(1 \%)$ & & \\
\hline Ruptured UPJ & & & & $1(1 \%)$ & & $1(0.3 \%)$ \\
\hline Perforated ureter & & & & $4(3.4 \%)$ & $2(3.2 \%)$ & $2(0.6 \%)$ \\
\hline Septic shock & & & & & & $2(0.6 \%)$ \\
\hline
\end{tabular}

$U P=$ upper pole $; M P=$ mid pole $[L P=$ lower pole $; U P J=$ ureteropelvic junction. 
Table 4 - Minor complications at various access sites.

\begin{tabular}{lcccccc}
\hline & Intercostal & Subcostal & & & & \\
\hline Location & UP & MP & LP & UP & MP & LP \\
No. Punctures & 111 & 12 & 4 & 119 & 63 & 333 \\
Complications & & & & $13(11 \%)$ & & $1(0.3 \%)$ \\
$\quad$ Atelectasis & $3(2.7 \%)$ & & & $2(1.7 \%)$ & $2(3.2 \%)$ & $13(3.9 \%)$ \\
Fever & $2(1.8 \%)$ & & $1(25 \%)$ & $3(2.5 \%)$ & $2(3.2 \%)$ & $15(4.5 \%)$ \\
Obstruction & $1(1 \%)$ & & $1(25 \%)$ & $2(1.7 \%)$ & $2(3.2 \%)$ & $8(2.4 \%)$ \\
Tract bleeding & $1(1 \%)$ & & & $1(1 \%)$ & $1(1.6 \%)$ & $3(0.9 \%)$ \\
RA/RV thrombus & & & & $2(1.7 \%)$ & $4(6.3 \%)$ & $1(0.3 \%)$ \\
Renal infarct & & & & & & \\
Blood transfusion & & & & & & \\
\end{tabular}

$U P=$ upper pole $; M P=$ mid pole $L P=$ lower pole $; A=$ renal artery; $R V=$ renal vein.

and nephrolithotomy in $64.5-98.3 \%$ of patients $(4,14,17,18)$. Conversely, extracorporeal nephrolithotripsy (SWL), even if complemented by follow-up medical management, achieves stone-free results in only about $37 \%$ of patients (19-21). Even in locations such as the lower pole, where SWL had been favored, PCNL rendered $90-95 \%$ of patients stone-free versus SWL (14-63\%) (19). Ureteroscopic nephrolithotripsy by electrohydraulic or Holmium Yag laser likewise cannot match PCNL results (22). For large stone loads $\left(2500 \mathrm{~mm}^{2}\right.$ or larger), staghorn calculi, calculi in diverticula and even smaller stones in lower pole calices; PCNL is now the preferred method. The choice of access tract is based on the ability to provide good visibility of the stone bearing area and a point of entry with minimal risk of injury to adjacent organs. Additionally, the access tract should provide a trajectory projecting without torque or angulation into the infundibulum and renal pelvis, hence facilitating atraumatic intraoperative advancement of the Amplatz sheath to the UPJ or inferior calyceal group (4,6,8,14,23; Figure-1). Intercostal access via the posterior superior calyx offers the best trajectory via infundibulum to pelvis, UPJ and inferior calyx $(4,10)$, Figure-2. Lack of angulation and torque when advancing the Amplatz sheath significantly reduces the risk of inducing bleeding. However, the preferred intercostal access route has been incriminated with a

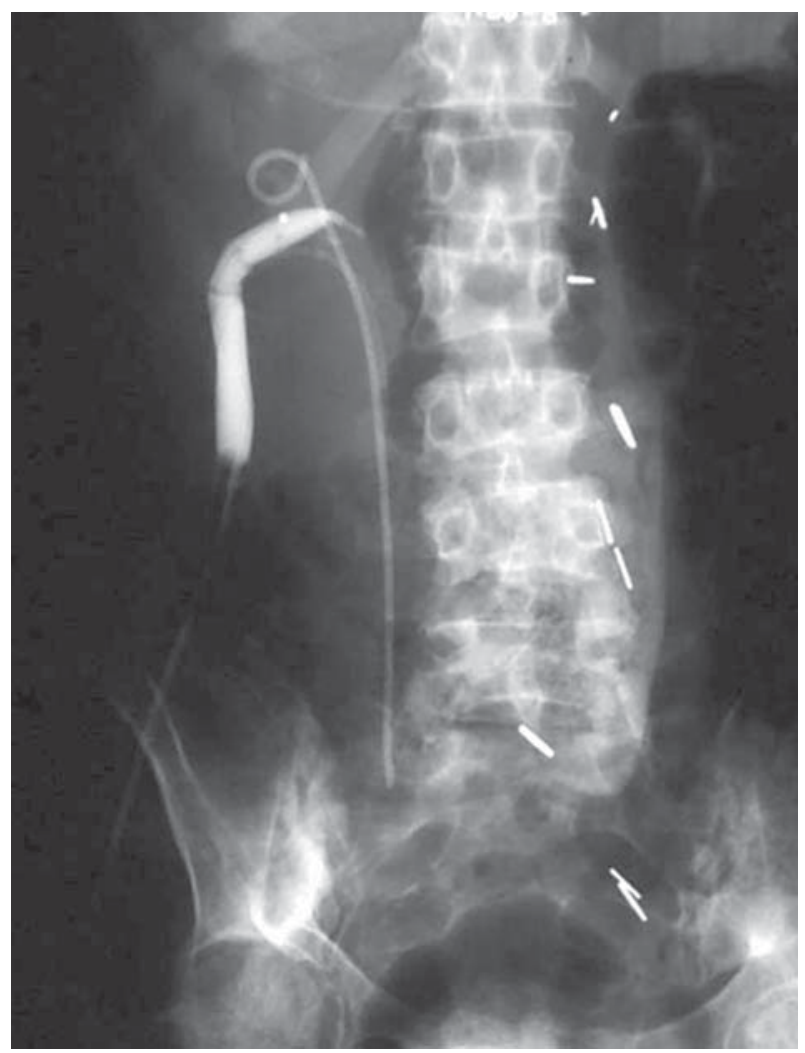

Figure 5 - A Kay tamponade balloon has been introduced into the access tract to curtail post procedural loosing. Note the acute angulation of the balloon reflecting the angle between the access tract and collecting system. Attempts to force an Amplatz sheath along such a tract causes injury to the peri-infundibular venous plexus and results in heavy bleeding. 
Table 5 - Complications attendant management of staghorn calculi.

\begin{tabular}{|c|c|c|c|c|c|c|}
\hline \multirow[b]{2}{*}{ Location } & \multicolumn{3}{|c|}{ Intercostal } & \multicolumn{3}{|c|}{ Subcostal } \\
\hline & UP & MP & LP & UP & MP & LP \\
\hline No. Punctures & 102 & 10 & 2 & 32 & 47 & 62 \\
\hline \multicolumn{7}{|l|}{ Complications } \\
\hline Pneumothorax & $1(1 \%)$ & & & $1(3.1 \%)$ & & \\
\hline Hemothorax & & & & & $1(2.1 \%)$ & \\
\hline Colo-caliceal fistula & & & & & $1(2.1 \%)$ & \\
\hline Arterio-caliceal fistula & $1(1 \%)$ & & & & & \\
\hline A-V Fistula & $2(2 \%)$ & & & & $1(2.1 \%)$ & \\
\hline Pseudoaneurysm & & & & $1(3.1 \%)$ & & \\
\hline Blood transfusion & & & & & $1(2.1 \%)$ & \\
\hline Ruptured UPJ & & & & $1(3.1 \%)$ & & \\
\hline Perforated ureter & & & & $1(3.1 \%)$ & $1(2.1 \%)$ & $1(1.6 \%)$ \\
\hline
\end{tabular}

$U P=$ upper pole $; M P=$ mid pole $; P=$ lower pole $; U P J=$ ureteropelvic junction.

higher rate of complications than the subcostal approach $(4,6,16,17)$. The supra 11 th rib approach has a particularly high rate of complications. Pneumoand hemothorax, and calyceal-pleural fistulae have been reported in up to $23.1 \%$ (4) The possibility of both a transthoracic and transpleural trajectory of this type of access tract, despite attempts to attain a high position of the lung by puncturing during the expiration phase, predisposes to these complications $(4,6,14,24)$. The incidence of hydropneumothorax occurring with intercostal access has been reported at a rate of $4 \%$ to $15.3 \%$, with subcostal access, $0 \%$ to $1.4 \%(4,7,14,16,24)$. Similarly, large pleural effusions were reported in $8 \%$ to $12.5 \%$ with intercostal approach, but virtually absent with subcostal access $(6,14,15,24)$. Moreover, on the basis of anatomic considerations, the intercostal access route might have a higher chance for injury to anterior segmental vessels or even anterior and posterior divisional arteries $(4,10,11,14)$ Figure-6.

In our series we encountered 4 major (3.6\%) and 7 minor complications (6.3\%) in 111 patients with intercostal access to the upper pole $(\mathrm{p}=0.0395$; Table4). Interestingly, there was only one pneumothorax $(0.9 \%)$. There were three vascular complications reflecting the inherent risk posed by anatomic proxim- ity of vascular and excretory system in the upper pole $(10,11)$. Both AV fistulae involved posterior division branches; the arterio-calyceal fistula was between a posterior middivisional branch and the posterior calyceal infundibulum. The etiology is most likely secondary to the needle penetrating the infundibulum and passing through the anteriorly located artery, giving rise to the fistula $(10,11)$. Only 7 minor injuries were recorded (Table-4). One Steinstrasse caused by multiple fragments in the lower ureter caused obstruction (Table-4). A renal branch vein thrombosis also resulted, without late sequellae. Three patients developed atelectasis on the ipsilateral side, likely related to irritation of the diaphragm and curtailed respiratory motion. Our incidence of intrathoracic complications $(3.1 \%)$ attendant to the access tracts compares favorably to that of the $7.1 \%-12 \%$ range reported in the literature $(4,6)$. Our complication rate for supra 11 th rib access is $25 \%$, similar to that of $23.1 \%$ of the literature $(6,16,24)$. Our technique of puncturing lateral to the erector muscle in mid expiration takes advantage of the higher location of the pleural deflection under these circumstances, a feature that has been pointed out by Hooper et al. (12).

In 119 subcostal access procedures to the upper pole, we experienced 9 major complications 

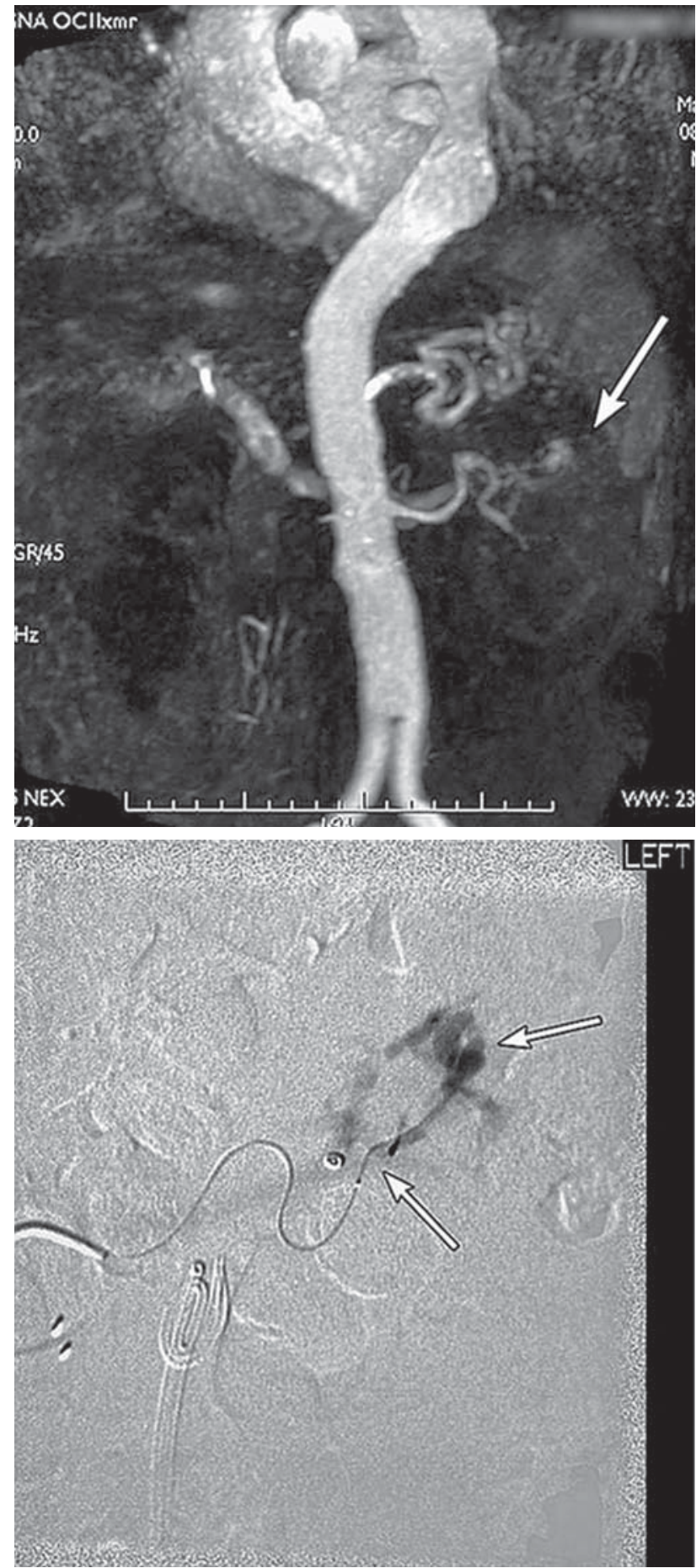

Figure 6 - A) Coronal maximum intensity projection of an abdominal MR angiogram demonstrating a post lithotripsy $A-V$ fistula of the left kidney (white arrow). B) A microcatheter has been advanced superselectively into the posterior divisional branch of the left renal artery feeding the A-V fistula (inferior arrow), which is occluded by two $2 \times 3 \mathrm{~mm}$ microcoils (superior arrow).
(Table-3). Pneumothoraces and rupture of UPJ and ureters likely occurred while manipulating and repositioning the Amplatz sheath during the stone extraction phase. Vascular injuries involving anterior divisional branches were also encountered, most likely secondary to excessive needle penetration. The large incidence of minor complications (22) deserves scrutiny (Table-4). Atelectasis in 13 patients suggests that the access route might have caused significant irritation of the diaphragm. Diffuse bleeding from the tract $(\mathrm{n}=2)$ and need for blood transfusions ( $\mathrm{n}$ $=2$ ) suggest either injury to the peri-infundibular plexus, either during the procedure or due to inadequate postoperative tamponade by the Malecot or Council Catheter. Injury to the peri-infundibular venous plexus is likely to occur when advancing the Amplatz sheath while negotiating the angle formed between the subcostal access tract and the infundibulum and pelvis $(8,11,15,16)$, (Figure-5). Access to the mid-calyx (interpolar calyx) via a subcostal approach shows a similar high incidence rate of minor complications (19\%), Table-4. The high incidence of diffuse tract bleed requiring transfusion is again attributable to injury of the peri-infundibular venous plexus. With access to the lower pole via subcostal route, diffuse tract bleeding, obstruction and fever are the most common minor complications (14), Table-5. Again, difficulty adjusting the Amplatz sheath interoperatively may result in injury, failure to completely evacuate stone debris (and hence Steinstrasse) and infection and fever. In the subgroup of staghorn calculi, the major complication rate for subcostal access is almost $10 \%$, but for intercostal access the rate is only 3\% (Table-5). This reflects the advantage of the intercostal approach when dealing with dendritic extension of stones.

We analyzed our data using logistic regression and predicted complications for the respective calyx and intercostal or subcostal entry. The complication rates for upper calyx were $17.3 \%$, for interpolar calyx $44.4 \%$, and for lower calyx $15.4 \%$. The odds of complication were 3.5 times higher for interpolar than upper polar entry $(p=0.0003) ; 5.1$ times higher for interpolar than lower polar entry $(p=0.0001)$. The odds of complications were 1.9 times higher with subcostal entry compared to intercostal entry ( $p$ $=0.0389$ ). 


\section{CONCLUSIONS}

The findings of our study support preferential use of intercostal access routes (12th, 11th, 10 th rib space) via the posterior calyx for percutaneous nephrolithotripsy in patients with a large stone load, staghorn calculi, multiple calculi lodged in the posterior superior calyx, pelvis, UPJ, upper ureter, and posterior and anterior inferior calyceal groups. This route offers optimal visibility, easy interoperative advancement and adjustment of the Amplatz sheath and rigid nephroscope, a low rate of procedural complications, reduced operative time and excellent results in removal of targeted stones. For calculi in other locations, separate access tracts or "Y" tracts are advocated.

\section{CONFLICT OF INTEREST}

None declared.

\section{REFERENCES}

1. Duvdevani M, Razvi H, Sofer M, Beiko DT, Nott L, Chew BH, et al.: Third prize: contemporary percutaneous nephrolithotripsy: 1585 procedures in 1338 consecutive patients. J Endourol. 2007; 21: 824-9.

2. Wolf JS Jr, Clayman RV: Percutaneous nephrostolithotomy. What is its role in 1997? Urol Clin North Am. 1997; 24: 43-58.

3. Osman MM, Alfano Y, Kamp S, Haecker A, Alken P, Michel MS, et al.: 5-year-follow-up of patients with clinically insignificant residual fragments after extracorporeal shockwave lithotripsy. Eur Urol. 2005; 47: 860-4.

4. Munver R, Delvecchio FC, Newman GE, Preminger GM: Critical analysis of supracostal access for percutaneous renal surgery. J Urol. 2001; 166: 1242-6.

5. Hentschel H, Janitzky V, Weirich T: Percutaneous nephrolithotomy - always effective and free of complications? Aktuelle Urol. 2007; 38: 232-6.

6. Radecka E, Brehmer M, Holmgren K, Magnusson A: Complications associated with percutaneous nephrolithotripsy: supra- versus subcostal access. A retrospective study. Acta Radiol. 2003; 44: 447-51.

7. Stening SG, Bourne S: Supracostal percutaneous nephrolithotomy for upper pole caliceal calculi. J Endourol. 1998; 12: 359-62.
8. Shaban A, Kodera A, El Ghoneimy MN, Orban TZ, Mursi K, Hegazy A: Safety and efficacy of supracostal access in percutaneous renal surgery. J Endourol. 2008; 22: 29-34.

9. Preminger GM, Schultz S, Clayman RV, Curry T, Redman HC, Peters PC: Cephalad renal movement during percutaneous nephrostolithotomy. J Urol. 1987; 137 : 623-5.

10. Sampaio FJ, Mandarim-De-Lacerda CA, De Aragão AH: The collector system of the kidney. Applied anatomy based on the analysis of 3-dimensional casts. J Urol (Paris). 1987; 93: 183-5.

11. Sampaio FJ, Aragao AH: Anatomical relationship between the intrarenal arteries and the kidney collecting system. J Urol. 1990; 143: 679-81.

12. Hopper KD, Sherman JL, Luethke JM, Ghaed N: The retrorenal colon in the supine and prone patient. Radiology. 1987; 162: 443-6.

13. Lojanapiwat B, Prasopsuk S: Upper-pole access for percutaneous nephrolithotomy: comparison of supracostal and infracostal approaches. J Endourol. 2006; 20: 491-4.

14. Yadav R, Aron M, Gupta NP, Hemal AK, Seth A, Kolla SB: Safety of supracostal punctures for percutaneous renal surgery. Int J Urol. 2006; 13: 1267-70.

15. Sukumar S, Nair B, Ginil KP, Sanjeevan KV, Sanjay $\mathrm{BH}$ : Supracostal access for percutaneous nephrolithotomy: less morbid, more effective. Int Urol Nephrol. 2008; 40: 263-7.

16. Lashley DB, Fuchs EF: Urologist-acquired renal access for percutaneous renal surgery. Urology. 1998; 51: 927-31.

17. Segura JW: Endourology. J Urol. 1984; 132: 107984.

18. Netto NR Jr, Ikonomidis J, Ikari O, Claro JA: Comparative study of percutaneous access for staghorn calculi. Urology. 2005; 65: 659-62; discussion 662-3.

19. Lam HS, Lingeman JE, Mosbaugh PG, Steele RE, Knapp PM, Scott JW, et al.: Evolution of the technique of combination therapy for staghorn calculi: a decreasing role for extracorporeal shock wave lithotripsy. J Urol. 1992; 148: 1058-62.

20. Fine JK, Pak CY, Preminger GM: Effect of medical management and residual fragments on recurrent stone formation following shock wave lithotripsy. J Urol. 1995; 153: 27-32; discussion 32-3.

21. Kang DE, Maloney MM, Haleblian GE, Springhart WP, Honeycutt EF, Eisenstein EL, et al.: Effect of medical management on recurrent stone formation following percutaneous nephrolithotomy. J Urol. 2007; 177: 1785-8; discussion 1788-9. 
22. Mariani AJ: Combined electrohydraulic and holmium: YAG laser ureteroscopic nephrolithotripsy of large (greater than $4 \mathrm{~cm}$ ) renal calculi. J Urol. 2007; 177: 168-73; discussion 173.

23. Aron M, Goel R, Kesarwani PK, Seth A, Gupta NP: Upper pole access for complex lower pole renal calculi. BJU Int. 2004; 94: 849-52; discussion 852.

\author{
Correspondence address: \\ Dr. Erich K. Lang \\ Department of Radiology \\ SUNY Downstate College of Medicine \\ 450 Clarkson Avenue \\ Box 1198, Brooklyn, NY, 11231, USA \\ Fax: + 1 718-270-3848 \\ E-mail: erich.lang@downstate.edu
}

\section{EDITORIAL COMMENT}

The mainstay of percutaneous nephrolithotomy is to create a straight path from the skin to the renal pelvis in order to avoid renal angulations and consequently damage to the peri-infundibular structures (either vessels or parenchyma).

Historically at the beginning of percutaneous renal surgery the patient was in prone position, to avoid colonic puncture, and the access was routinely subcostal, to avoid injury to the lungs and pleura, but through the lower calyx $(1,2)$. However, it has also been recognized that, in selected cases, it could not provide an optimal access. In this retrospective analysis on a quite large series of percutaneous nephrolithotomy (PCNL), Dr. Lang and co-authors address the so-called "intercostal (or supracostal)" approach for PCNL. This issue is still highly debated in the field of endourology, even if its related literature remains scarce. Unfortunately, the value of this report is negatively affected by some major drawbacks.

First, all the inherent biases of a retrospective study are present here and this should be taken into account when looking at the conclusions.
24. Narasimham DL, Jacobsson B, Vijayan P, Bhuyan BC, Nyman U, Holmquist B: Percutaneous nephrolithotomy through an intercostal approach. Acta Radiol. 1991; 32: 162-5.

Accepted after revision:

January 6, 2009
One of the significant evolutions of the PCNL has been the widespread-though not universal-renal puncture by a urologist, making it a single-stage procedure (3). Here, PCNL is presented as a two-step procedure, the first in local anesthesia, performed by the radiologist, the second in general anesthesia, performed by the urologist.

The authors emphasize the benefits of the upper pole access and we can agree with them that it allows a good exposure of most of the calyces and of the proximal ureter. As the main requirement during PCNL is always the same, a straight path causing no angulations, if the superior calyx is too high, intrathoracic, they should not perform a subcostal puncture: a complication will almost certainly occur.

However, many of the complications reported are related to the lithotripsy rather than to the puncture. The high rate of ureteral perforation and UPJ avulsion are not puncture-related but a matter of a wrong operative technique.

It has been previously suggested that a great difference might be between the supra twelfth rib ap- 
proach, which is transthoracic but extrapleural, and the supra eleventh rib access, which is both transthoracic and transpleural. This issue is not addressed in the paper (4).

To note that the authors do not use the insertion of a ureteral catheter as a first step of the procedure (as most of us performing PCNL routinely do). The needle is inserted and the contrast medium is injected without a prior retrograde dilation of the calyceal system. With this technique, if you do not correctly target the calyx the risk of fornix rupturing during the path dilation and subsequent bleeding is increased.

Finally, we would like to remind the authors that supine position for PCNL has been advocated in the past decade (5). One of its main advantages is that it might combine the benefits of percutaneous and ureteroscopic intrarenal surgery in selected cases (6). Thus, large and/or complex urolithiasis can be treated with a high one-step stone-free rate, unquestionable anesthesiological advantages, and no additional procedure-related complications (7). In this regard, some limitations of the standard prone PCNL might be overcome avoiding a potentially harmful supracostal approach, as the one proposed.

\section{REFERENCES}

1. Alken P, Hutschenreiter G, Günther R, Marberger M: Percutaneous stone manipulation. J Urol. 1981; 125 : 463-6.

2. Gupta R, Kumar A, Kapoor R, Srivastava A, Mandhani A: Prospective evaluation of safety and efficacy of the supracostal approach for percutaneous nephrolithotomy. BJU Int. 2002; 90: 809-13.

3. Smith AD: Percutaneous punctures--is this the endourologist's turf? J Urol. 1994; 152: 1982-3.

4. Munver R, Delvecchio FC, Newman GE, Preminger GM: Critical analysis of supracostal access for percutaneous renal surgery. J Urol. 2001; 166: 1242-6.

5. Valdivia Uría JG, Valle Gerhold J, López López JA, Villarroya Rodriguez S, Ambroj Navarro C, Ramirez Fabián M, et al.: Technique and complications of percutaneous nephroscopy: experience with 557 patients in the supine position. J Urol. 1998; 160: 1975-8.Autorino R, Giannarini G: Prone or supine: is this the question? Eur Urol. 2008; 54: 1216-8.

6. Scoffone CM, Cracco CM, Cossu M, Grande S, Poggio M, Scarpa RM: Endoscopic combined intrarenal surgery in galdakao-modified supine valdivia position: a new standard for percutaneous nephrolithotomy? Eur Urol. 2008; 54: 1393-403.

\section{Dr. Riccardo Autorino \& Dr. Marco De Sio Division of Urology Second University of Naples Naples, Italy E-mail:ricautor@tin.it}

\section{EDITORIAL COMMENT}

The authors compare a series of patients with percutaneous nephrolithotripsy (PCNL) tracts above the 12th and 11th ribs to a group with access established in a subcostal location. Complications including those related to the thorax are no greater when the access is an intercostal upper pole approach. We as well have seen a shift to an upper pole approach over the last several years and this is now at least as common as a lower pole approach at our centre (1). For these reasons the authors emphasize including less torquing of the working sheath, working "downhill" on most large stones and ready access to more of the collecting system. They do not mention hydrothorax as a specific thoracic complication however this may be a more common adverse consequence of a high approach as pneumothorax. Attention to maintaining the working sheath in the collecting system throughout the procedure is an important technical point. We also are more likely to place a stent at the conclusion of the PCNL procedure with a supracostal access to assure 
antegrade urine drainage postoperatively and prevent fluid accumulation in the chest. In such instances, it is important to remove the urethral catheter after the percutaneous tube is removed to prevent retrograde extravasation of urine.

Access in this series was obtained in the radiology department. I believe that similar results with a low rate of thoracic complications can be achieved when the urologist obtains the percutaneous access in the operative room using $\mathrm{C}$-arm fluoroscopic guidance (2).

Finally, flexible nephroscopy combined as needed with intracorporeal lithotripsy is an important adjunct to minimize the need for additional tracts when performing PCNL.

\section{REFERENCE}

1. Duvdevani M, Razvi H, Sofer M, Beiko DT, Nott L, Chew BH, et al.: Third prize: contemporary percutaneous nephrolithotripsy: 1585 procedures in 1338 consecutive patients. J Endourol. 2007; 21: 824-9.

2. Watterson JD, Soon S, Jana K: Access related complications during percutaneous nephrolithotomy: urology versus radiology at a single academic institution. J Urol. 2006; 176: 142-5.

Dr. John Denstedt Richard Ivey Professor and Chair Department of Surgery The University of Western Ontario London, ON, Canada

E-mail: john.denstedt@sjhc.london.on.ca 